\title{
Granulomatous inflammation of gall bladder mimicking an impacted common bile duct stone
}

\author{
Muhammad Saad Faisal, Sidra Dil Muhammad \\ Sharif Medical \& Dental College, Raiwind Road, Lahore, Pakistan
}

Keywords: Granulomatous inflammation; tuberculosis; gall bladder; impacted CBD stone

\section{Introduction}

Although intrabdominal tuberculosis is common in developing countries (reported incidence is $12 \%$ ), it rarely involves the biliary system [1]. Clinical presentation is usually nonspecific [1-3]. Radiological findings of the gallbladder may raise the suspicion of malignancy [1]. Diagnosis is often made postoperatively on histopathology examination $[4,5]$. Herein, we present a case of a young male who presented with recurrent right upper abdominal pain, treated as a case of impacted CBD stone, was incidentally diagnosed as tubercular cholecystitis.

\section{Case presentation}

A 29 years old male (non-diabetic, non-smoker), was admitted for management of acute calculus cholecystitis at Rasheed Hospital, Lahore though A\&H Laparoscopic Clinic, Lahore in July 2017. Mildly deranged liver enzymes and normal TLC $(9.4 \times 103 \mu \mathrm{L})$ were recorded at time of admission. An edematous gall bladder with a wall thickness (up to $1 \mathrm{~cm}$ ), multiple heterogenic foci in the fundus of the gall bladder and normal-sized CBD were observed on ultrasonography. For further elaboration of heterogenic foci in the gall bladder, CT scan performed that reported a hypoechoic mass within the fundus of gall bladder surrounded by a rim of inflammatory exudate and a normalsized CBD with small heterogenous shadows in its wall. The patient responded well to antibiotic therapy and discharged from hospital with a plan of interval cholecystectomy.

A preoperative ultrasound showed edematous thick wall $(9 \mathrm{~mm})$ gall bladder containing debrinous fluid around a perforated area and single stone like a shadow (measuring $5 \mathrm{~mm}$ ) in CBD with proximal dilatation. Liver function tests were within the normal range. MRCP confirmed the gall bladder wall thickness of up to $8 \mathrm{~mm}$, multiple calculi in the gall bladder and $6 \mathrm{~mm}$ sized CBD that had an impacted non

Correspondence: Muhammad Saad Faisal

E-mail: ahlaparoscopicclinic@gmail.com

(i) https://orcid.org/0000-0002-7972-0072

Received: 30-12-2019 Accepted: 15-06-2020

DOI: http://doi.org/10.4038/sljs.v38i2.8655

The Sri Lanka Journal of Surgery 2020; 38(2): 75-77

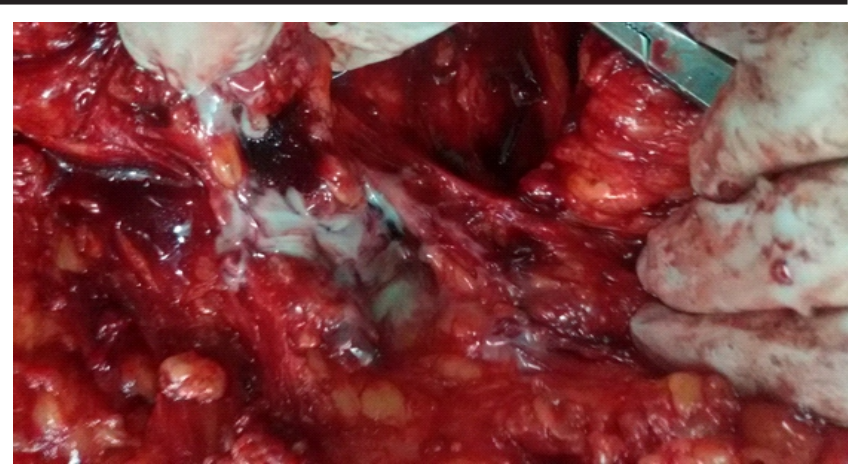

Figure 1. An operative view: Pus oozing out from thick walled shrunken gall bladder wall

obstructing shadow in its wall most likely a stone. Pancreatic parenchyma and the pancreatic duct were also normal. ERCP demonstrated normal CBD with no intraluminal pathology. An open cholecystectomy with CBD exploration was planned. Per-operatively, dense adhesions between the gall bladder and omentum were encountered. Meticulous dissection through adhesions explored a thickened, edematous gall bladder that oozing out the cheesy material and pus from its indurated areas while attempting to grasp the gall bladder with help of grasper (Figure 1).

Perforation at the fundus of the gall bladder was revealed. Due to dense adhesions, we could not negotiate the dissection of the calot's triangle. On opening the gall bladder, few calculi in the lumen and jam-packed stones in different compartments of gall bladder were noticed. Narrow gall bladder lumen was traced towards CBD. A sub centimetre sized tubercle was palpable on CBD near the cystic duct area which raised the suspicion of impacted stone.

A longitudinal incision was made over it aiming to extract the stone however, cheesy material oozed out. No intramural stone was found on CBD exploration. Per-operative cholangiogram excluded any stone presence and the dye was freely inflowing in the duodenum.

The procedure was completed with the placement of T-tube and subhepatic drain. Histopathology of resected specimen reported the presence of multiple scattered granulomas composed of a collection of epithelioid, histiocytes with admix lymphocytes and plasma cells. The granuloma 


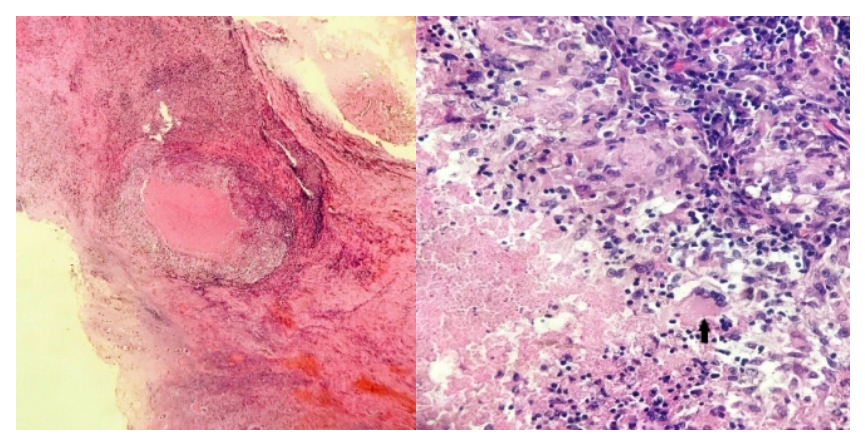

Figure 2. : A: Scanner view of gallbladder wall section with an ulcerated lining and a granuloma with central necrosis. (H\&E stain, 4x magnification), B: High power field of granuloma showing epithelioid cells, necrosis and multinucleated giant cells (black arrow). (H\&E stain, 40x magnification)

containing multiple Langerhans type giant cells containing multiple nuclei giving the horseshoe-shaped appearance, associated caseous necrosis was seen (Figure 2).

A tuberculin test was also found to be positive. The patient recovered completely without any significant complication. He showed improvement with the antituberculous treatment within 30 days interval. He gained weight up to $2 \mathrm{Kg}$. The patient was followed up for 2 years. He remained symptoms free.

\section{Discussion}

Gallbladder tuberculosis is more common in males (M: F; $2: 1)$ with an age range of 30-50 years [1,2]. Hepatobiliary tuberculosis constitutes less than $1 \%$ of the total abdominal tuberculosis cases [3]. Low incidence of gallbladder tuberculosis is largely attributed to the high content of bile, alkaline $\mathrm{pH}$ and other inhibitory substances [4,5]. Tubercular infection may spread to gallbladder by peritoneal, lymphatic, hematogenous or ascending routes [5].

Most of the patients present with pain abdomen, anorexia and weight loss 1.5. Unusual presentation in the form of abdominal lump, perforation with abscess formation in anterior abdominal wall and port site sinus formation has also been described [2].

Radiological findings demonstrate cholelithiasis, wall thickening or intraluminal mass simulating gallbladder malignancy [6]. Laboratory findings include anaemia, raised ESR and positive tuberculin test. AFB examination of bile obtained from ERCP has extremely low sensitivity, however, increased adenosine deaminase (ADA) level favours tubercular aetiology. In our case, the clinical presentation was very misleading.
At initial presentation, both clinical and radiological findings were favouring acute cholecystitis. At 6-week interval, radiological findings still showed thick-walled gall bladder with an impacted CBD stone, which was further confirmed on MRCP and ERCP. But upon exploration, it was found to be a tuberculous lesion and histopathology confirmed the diagnosis. Theoretically, a preoperative diagnosis of tuberculosis may be established by brush cytology on ERCP, and PCR of bile for tuberculosis. However, it is not considered routinely. Reportedly, this is the first case of tuberculous lesion mimicking an impacted stone in CBD. Previously, some cases of tuberculosis of CBD causing stricture or obstruction has been reported [5].

This disease needs differentiation from acute and chronic cholecystitis including xanthogranulomatous cholecystitis, carcinoma and polypoidal lesions of gallbladder [4]. Regional lymphnodal enlargement is seen in both gallbladder tuberculosis and malignancy. Liver infiltration or metastasis is seen in gallbladder malignancy while lung lesions, omental or mesenteric thickening is frequent in tuberculosis. Treatment protocol like abdominal tuberculosis includes initial four drugs intensive phase for two months and two drugs for the continuation phase [5].

Ahmed $\mathrm{HG}$ et al, in a retrospective descriptive study, investigated the morphological pattern of tuberculous lymphadenitis and reported that the most major histological features were giant cell (88\%), caseation (84\%), epithelioid cells $(80 \%)$, granuloma and caseation (68\%), lymphocytes (31\%), and histiocytes (4\%) [6].

In this case, histopathology of diseased gall bladder showed mandatory histological features of tuberculosis to initiate anti-tuberculous treatment. The polymerase chain reaction is a reliable and rapid test for the detection of Mycobacterium tuberculosis (sensitivity 78.3\%) [7]. However, we didn't perform PCR or AFB before the start of antituberculous drugs as we had histopathological confirmation of tuberculosis.

\section{Conclusion}

Gallbladder tuberculosis being a very uncommon entity needs consideration in cases of cholecystitis and gallbladder mass, particularly in endemic areas. The high degree of clinical suspicion and mandatory histopathological examination is essential for diagnosis and management of gallbladder tuberculosis.

All authors disclose no conflict of interest. The study was conducted in accordance with the ethical standards of the relevant institutional or national ethics committee and the Helsinki Declaration of 1975, as revised in 2000 . 


\section{References}

1. Liu Y, Wang K, Liu H. Gallbladder tuberculosis mimicking gallbladder carcinoma: a case report and literature review. Case reports in hepatology. 2016;2016. https://doi.org/10.1155/2016/3629708

2. Butt UI, Hameed B, Farooka MW, Ayyaz M, Chughtai A. Tuberculosis of Gallbladder mimicking carcinoma. Journal of the College of Physicians and Surgeons Pakistan 2017, Vol. 27 (Special Supplement 2 of Case Reports): S84-S85.

3. Xu XF, Yu RS, Qiu LL, Shen J, Dong F, Chen Y. Gallbladder tuberculosis: CT findings with histopathologic correlation. Korean J Radiol 2011;12(2):196-202. https://doi.org/10.3348/kjr.2011.12.2.196

4. Krishnamurthy G, Singh H, Rajendran J, Sharma V, Yadav TD, Gaspar BL, Vasishta RK, Singh R. Gallbladder tuberculosis camouflaging as gallbladder cancer-case series and review focussing on treatment. TAI 2016;3(6):152-7.

https://doi.org/10.1177/2049936116678589

5. Sumantra Ray, Sajib Chatterjee, Asis Kumar Saha, and Saugata Samanta. Obstructive Jaundice Due to Tuberculosis of Distal $\mathrm{CBD}$ and Periampullary Region Mimickcholangiocarcinoma. Niger J Surg. 2012 Jan-Jun; 18(1): 17-18.

6. Ahmed HG, Nassar AS, Ginawi I. Screening for tuberculosis and its histological pattern in patients with enlarged lymph node. Pathol Res Int 2011;2011. https://doi.org/10.4061/2011/417635

7. Cheng VC, Yam WC, Hung IF, Woo PC, Lau SK, Tang BS, Yuen KY. Clinical evaluation of the polymerase chain reaction for the rapid diagnosis of tuberculosis. J Clin Pathol 2004;57(3):281-5. https://doi.org/10.1136/jcp.2003.012658

\section{Learning Points:}

- Very rarely seen pathology, tuberculosis of hepatobililary tract, has no pathognomonic clinical or radiological features which makes it almost impossible to diagnose preoperatively.

- The only chance of establishing diagnosis is histopathological evidence which is mandatory in every hepatobiliary surgery.

- Still there is paucity of data to establish any diagnostic or screening criteria even in endemic areas of the world.

- Adequate resection and routine anti tuberclous treatment regimens may provide cure without significant morbidity. 